\section{Preface: Happy 10th anniversary!}

\author{
Olivera J. Finn
}

Published online: 30 June 2011

(C) Springer Science+Business Media, LLC 2011

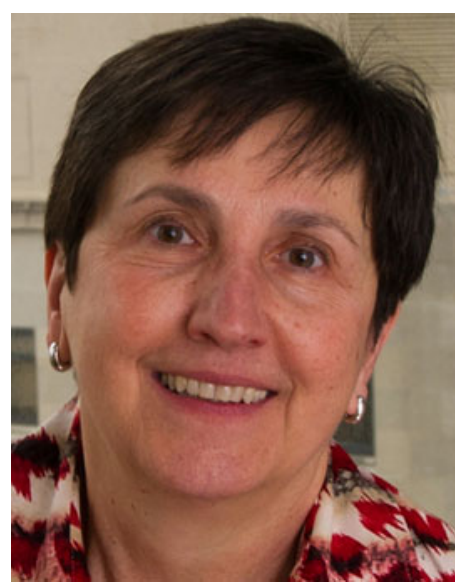

Olivera J. Finn, PhD

Distinguished Professor and Chair
On January 1, 2012, University of Pittsburgh School of Medicine Department of Immunology will celebrate its 10th anniversary. This may come as a surprise to many of our colleagues around the world who are familiar with the long track record of accomplishments of Pittsburgh immunologists in the fields of vaccines, transplantation immunology, autoimmunity, and cancer immunology that reaches much further back than 10 years. Immunology, as a relatively young discipline, was until 2001 practiced at Pitt in basic science departments organized around the more traditional and more established disciplines and in several enlightened clinical departments that early on recognized the importance of this new science in health and disease. The Nobel Prize-winning immunologist Neils Jerne studied antibody-producing cells and developed the Jerne Plaque Assay in the Department of Microbiology that he chaired from 1962 to 1966. In 1947, Jonas Salk came to Pittsburgh and labored with his team in a small virology laboratory from which would emerge a vaccine against polio. The Tom E. Starzl Transplant Institute celebrated this year the 30th anniversary of the arrival of this famous transplant surgeon to Pittsburgh in 1981, where in the Department of Surgery chaired by a surgeon/immunologist Richard Simmons, some of the best transplantation immunology research was performed in support of clinical transplantation. Outstanding immunologists were making groundbreaking discoveries about HLA and diabetes in the Department of Pediatrics and others were addressing the new puzzle that was AIDS in our highly ranked School of Public Health. Cancer immunologists started coming to

\section{O. J. Finn $(\bowtie)$}

Department of Immunology, University of Pittsburgh School of Medicine, Pittsburgh, PA, USA

e-mail: ojfinn@pitt.edu
Pittsburgh in 1985 with the arrival of a famous immunologist Ronald Herberman who founded the Pittsburgh Cancer Institute with Immunology as one of its leading programs.

Because the blood and soul of our research enterprise are our students, Pittsburgh immunologists first united under the auspices of the $\mathrm{PhD}$ granting Immunology Training Program that saw its first student graduate in 2000 with the first $\mathrm{PhD}$ in Immunology ever awarded at this university. Even though many students were previously trained here in immunology, the names of their degrees carried the names of the primary departments of their mentors.

The opportunity to fully recognize the excellence in immunology, and to ensure that it continues at the University of Pittsburgh as an independent and mature scientific discipline, came with the arrival in 1998 of Arthur S. Levine to the position of the Senior Vice Chancellor of the Schools of Health Sciences. He saw the need and the potential to renew the vigor of our research enterprise and he went about it by establishing new basic science departments and recruiting new world-renowned faculty to fill them. Department of Immunology was the first, followed by the Departments of Computational and Systems Biology, Structural Biology, and Developmental Biology. With the preexisting Departments of Microbiology and Molecular Genetics, Pharmacology and Chemical Biology, and Neurobiology, the environment for doing modern immunology research could not have been any better. Various centers and institutes also sprung up such as Drug Discovery Institute, Center for Vaccine Research, and Institute for Regenerative Medicine. Each has among its members immunology faculty and each features and supports immunology research. University of Pittsburgh is 5th in total grant funding from the National Institutes of Health 
and many individual grants, program projects, and center grants led by immunologists contribute to this high rank.

Department of Immunology started with 5 primary and 15 secondary faculty members, and on its 10th birthday, it numbers 15 primary and 57 secondary faculty members from most of the departments in the School of Medicine and the School of Arts and Sciences. We have a healthy mix of PhDs, MDs, and MD/PhDs. We carry out research, publish, and train our students in basic immunology, autoimmunity, cancer immunology, and transplantation immunology. We are actively involved in teaching medical students, graduate students, and undergraduate students.
We participate actively in many peer review activities and we and our trainees are active members of many professional organizations, most importantly The American Association of Immunologists.

In this issue, which fortuitously came to be on the eve of our 10th anniversary, we elected to feature projects that go from very basic to fully translational immunology. This type of research is only possible in communities like ours where basic scientists work shoulder to shoulder with clinical scientists and where over the years we have learned to speak each others language. 